\title{
Necessidades energéticas e consumo alimentar de adolescentes do interior baiano
}

\author{
Energetic needs and food consumption of adolescents from municipalities of Bahia \\ Necesidades energéticas y consumo alimentario de adolescentes del interior Bahía
}

Recebido: 2510/2021 | Revisado: 02/11/2021 | Aceito: 03/11/2021 | Publicado: 06/11/2021

Tailane dos Santos Assunção

ORCID: https://orcid.org/0000-0002-1806-0752

Universidade Federal da Bahia, Brasil

E-mail: tailane.ass@gmail.com

Rosemary da Rocha Fonseca Barroso

ORCID: https://orcid.org/0000-0003-3427-3288

Universidade Federal da Bahia, Escola de Nutrição, Brasil E-mail: rosefonseca@ufba.br

Ingrid Cardoso Fideles

ORCID: https://orcid.org/0000-0001-8358-3747 Universidade Federal da Bahia, Escola de Nutrição, Brasil E-mail: ingridcf@ufba.br

Rosana Aquino

ORCID: https://orcid.org/0000-0003-3906-5170 Universidade Federal da Bahia, Instituto de Saúde Coletiva, Brasil E-mail: aquino@ufba.br

\begin{abstract}
Resumo
Com o objetivo de contribuir com uma abordagem metodológica, este estudo comparou as necessidades energéticas, obtidas por meio de equações preditivas, com os dados de consumo alimentar de uma população de adolescentes escolares, de municípios baianos, e ainda, conhecer o espaço alimentar e as refeições realizadas por essa população. Trata-se de um estudo transversal, aninhado a um ensaio comunitário randomizado controlado, realizado com estudantes do ensino médio de escolas públicas de dez municípios baianos. O consumo alimentar foi obtido por meio de questionário de frequência alimentar quantitativo e de três registros alimentares. Utilizaram-se as Dietary Reference Intakes e Schofield para o cálculo das necessidades nutricionais. Dados antropométricos (peso, altura, circunferência da cintura), sociodemográficos e de atividade física foram analisados de forma descritiva e associações testadas usando o teste do qui-quadrado e teste exato de Fischer, com nível de significância de 0,95. A amostra foi composta por 85 adolescentes, eutróficos $(83,1 \%)$, ativos $(69,5 \%)$, e a maioria das refeições principais (café da manhã, almoço e jantar) sendo realizadas no domicílio (>80\%). As diferenças entre as necessidades nutricionais recomendadas e o consumo alimentar, foram estatisticamente significativas $(\mathrm{p}<0,05)$ e sinalizam a necessidade da acurácia na investigação de desfechos relacionados à alimentação e gasto energético em adolescentes. A avaliação das necessidades nutricionais para grupos populacionais é de importância no âmbito da Saúde Pública, bem como, conhecer a situação alimentar e nutricional preponderante em todas as faixas etárias, especialmente nos estágios de vida críticos para a formação de novos hábitos, como a adolescência.
\end{abstract}

Palavras-chave: Adolescentes; Comportamento alimentar; Necessidades nutricionais; Consumo calórico; Atividade física.

\begin{abstract}
In order to contribute with a methodological approach, this study compared the energy needs by predictive equations, with food consumption data from a population of school adolescents, from Bahia municipalities, and also to know the food space and the meals included by this population. This is a cross-sectional study, nested in a randomized controlled trial carried out with high school students from public schools in ten cities in Bahia. Food consumption was established through a quantitative food frequency questionnaire and three food records. They were used as Dietary Reference Intakes and Schofield to calculate nutritional needs. Anthropometric (weight, height, waist circumference), sociodemographic and physical activity data were carried over descriptively and associations were tested using the chisquare test and Fisher's exact test, with a significance level of 0.95 . The sample consisted of 85 adolescents, eutrophic $(83.1 \%)$, active $(69.5 \%)$, and most main meals (breakfast, lunch and dinner) were taken at home $(>80 \%)$. The differences between the recommended nutritional needs and food consumption were statistically relevant $(\mathrm{p}<0.05)$ and indicate the need for accuracy in the investigation of outcomes related to food and energy expenditure in adolescents. The assessment of nutritional needs for population groups is important in the context of Public Health, as well as knowing the prevailing food and nutritional situation in all age groups, especially in critical life stages for the formation of new habits, such as adolescence.
\end{abstract}

Keywords: Adolescent; Feeding behavior; Nutritional requirements; Eating; Exercise. 


\begin{abstract}
Resumen
Con el fin de contribuir con un enfoque metodológico, este estudio comparó la necesidad energética, através de ecuaciones predictivas, con datos de consumo de alimentos de una población de adolescentes escolares, de los municipios de Bahía, y para conocer el espacio alimentario y las comidas incluidas por esta población. Se trata de un estudio transversal, anidado en un ensayo controlado aleatorizado con estudiantes de secundaria de escuelas públicas en diez ciudades de Bahía. El consumo de alimentos se estableció mediante un cuestionario de frecuencia alimentaria y tres registros alimentarios. Se utilizaron como Ingestas Dietéticas de Referencia y Schofield para calcular las necesidades nutricionales. Los datos antropométricos, sociodemográficos y de actividad física se trasladaron de forma descriptiva y se probaron las asociaciones mediante la prueba de chi-cuadrado y exacta de Fisher, con nivel de significancia de 0,95. La muestra estuvo formada por 85 adolescentes, eutróficos $(83,1 \%)$, activos $(69,5 \%)$ y la mayoría de las comidas principales (desayuno, almuerzo y cena) se tomaban en casa (> 80\%). Las diferencias entre las necesidades nutricionales recomendadas y el consumo de alimentos fueron estadísticamente relevantes $(\mathrm{p}<0,05)$ e indican la necesidad de precisión en la investigación de los resultados relacionados con el gasto de alimentos y energía en adolescentes. La evaluación de las necesidades nutricionales de los grupos de población es importante en el contexto de la Salud Pública, así como conocer la situación alimentaria imperante en todos los grupos de edad, especialmente en etapas vitales críticas para la formación de nuevos hábitos, como la adolescencia.
\end{abstract}

Palabras clave: Adolescente; Conducta alimentaria; Necesidades nutricionales; Ingestión de alimentos; Ejercicio físico.

\title{
1. Introdução
}

As intensas modificações econômicas, sociais e culturais que vêm ocorrendo no País, nas últimas décadas, promoveram alterações nos hábitos e comportamentos alimentares, por exemplo, o aumento no consumo alimentar fora de casa (De Souza \& Enes, 2013) assim como, do consumo de alimentos processados e a substituição das refeições e preparações tradicionais por lanches de alta densidade energética, elevado conteúdo de açúcares e sódio e baixo teor de fibras e micronutrientes, constituindose como um importante fator de risco para o aumento da prevalência de obesidade e de Doenças Crônicas Não Transmissíveis (DCNT) (Monteiro et al., 2017), conforme evidenciado pela última Pesquisa de Orçamentos Familiares (POF) (Instituto Brasileiro de Geografia e Estatística, 2011).

Esse cenário atinge aos diversos ciclos da vida, em especial, a adolescência que é uma fase caracterizada por transformações vitais, biológicas e sociais, especialmente as de natureza comportamentais, como por exemplo, a exposição ao álcool, tabaco, drogas ilícitas, sedentarismo e a comportamentos alimentares considerados pouco saudáveis (World Health Organization, 2021). O adolescente passa por uma etapa crítica para construção de sua personalidade e identidade, bem como de sua inserção no meio social em patamares radicalmente distintos daqueles experimentados na infância (World Health Organization, 2013; De Souza \& Enes, 2013) e as escolhas alimentares e preferências deste grupo, ganham prioridade sobre os hábitos adquiridos em família. Esse padrão de consumo vai contribuir para caracterizar sua saúde, crescimento e desenvolvimento (Marchioni, Gorgulho, Teixeira, Verly Junior \& Fisberg, 2015).

Em relação à alimentação, diversos estudos evidenciaram discrepâncias entre as recomendações diárias de consumo dos alimentos protetores e os padrões alimentares adotados por esse grupo populacional, refletidas também pela alta frequência de consumo de alimentos relacionados a risco alimentar (Monge-Rojas, 2001; Barquera, et al., 2008; Levy et al., 2010; Leal, Philippi, Matsudo \& Toassa, 2010; Instituto Brasileiro de Geografia e Estatística, 2011; Santos, Fiaccone, Barreto, Silva \& Silva, 2014).

Entre os adolescentes, o tempo gasto em comportamentos sedentários (CS), resultado do tempo dedicado à prática de atividade física e ou atividades sedentárias contemporâneas (como assistir televisão, usar jogos eletrônicos, celulares, e ficar mais tempo sentado) frequentemente é encontrado no Brasil (Oehlschlaeger, Pinheiro, Horta, Gelatti, \& San'Tana, 2004; Farias Júnior et al., 2009), e em outros países (Ambrosini et al., 2009; del Mar Bibiloni, Pich, Córdova, Pons, \& Tur, 2012), e vêm crescendo ao longo do tempo (Costa et al., 2018). A combinação entre baixos níveis de atividade física (AF), altos níveis de comportamento sedentário e inadequados hábitos alimentares está associada a uma série de desfechos desfavoráveis à saúde do adolescente, como obesidade e fatores de risco cardiovasculares (World Health Organization, 2013). 
Associadas às mudanças nos hábitos alimentares na adolescência, têm-se as demandas nutricionais que são caracterizadas por aumento das necessidades de energia e nutrientes. Essa conjuntura pode incentivar diferentes tipos de distúrbios relacionados à nutrição (Moreno et al., 2014). Existem evidências, na literatura científica internacional, que a adoção precoce de hábitos saudáveis na infância e adolescência está relacionada com a permanência dos mesmos na fase adulta (Lake, Mathers, Rugg-Gunn \& Adamson, 2006; Berz, Singer, Guo, Daniels \& Moore, 2011; World Health Organization, 2013) e, consequentemente, com efeitos na redução da prevalência de problemas de saúde e na melhoria da qualidade de vida dos indivíduos.

A alimentação e nutrição integram requisitos básicos para a promoção e a proteção da saúde, assegurando a afirmação plena do potencial de crescimento e desenvolvimento humano, com qualidade de vida e cidadania (Política Nacional de Alimentação e Nutrição, 2013). E a alimentação escolar, operacionalizada através do Programa Nacional de Alimentação Escolar (PNAE), constitui-se como uma prática alimentar essencial nas escolas públicas brasileiras de ensino fundamental e médio, pois o ato de comer se realiza em meio a outras práticas que compõem o espaço escolar, caracterizando-se em experiências e processos que intervém na construção de hábitos alimentares e de identidades dos adolescentes (Silva, Amparo-Santos \& Soares, 2018), influenciadas pela rede formada na escola, na família e em outros espaços sociais.

Para identificação do perfil de consumo alimentar desse grupo populacional pode-se utilizar diferentes métodos, como a história dietética, registro alimentar, recordatório alimentar de 24 horas (R24h) e questionário de frequência alimentar (QFA), os quais são utilizados para avaliar a ingestão dietética (Schneider et al., 2016). O QFA permite estimar o consumo alimentar habitual e categorizar os indivíduos de acordo com diferentes níveis de consumo e proporciona estimativas das medidas de associação entre os fatores da dieta e a ocorrência de doenças (Brito, Araújo, Guimarães \& Pereira, 2017). Porém, pode apresentar alguns vieses de informação, como o de memória, levando a uma superestimação ou subestimação do consumo alimentar pelos entrevistados (Voci, Enes \& Slater, 2008; Neves, Gonzaga, Martens \& Moura, 2010), ocultando possíveis deficiências de nutrientes. Já o registro alimentar (RA) é um método onde o preenchimento dos alimentos e bebidas ingeridas, assim como suas quantidades, é feito pelo próprio indivíduo durante um período de tempo pré-estabelecido, e é um instrumento bastante válido para mensurar a ingestão ainda que também apresente algumas desvantagens como possíveis omissões de alimentos e erros na mensuração das porções, vêm sendo usado como instrumento de referência para avaliação do consumo alimentar de adolescentes (Mascarenhas et al., 2016).

Outra importante questão recai sobre os métodos para mensurar o gasto energético dos diferentes grupos populacionais. Existem dificuldades para se identificar um único método com validade, fidedignidade e facilidade de uso, que possa ser empregado rotineiramente em pediatria. Na ausência desse tipo de método, Vasconcelos, Barbosa, Pinto, Lima \& Araújo, (2011) recomendam a utilização de equações preditivas das necessidades energéticas, que são métodos rápidos e fáceis, além de terem baixo custo.

Dentre estas equações, têm-se a proposta das Dietary Reference Intakes - DRIs (Institute of Medicine, 2002/2005) para se obter as estimativas das necessidades de energia, utilizando-se o EER (Em inglês: Estimated Energy Requiriment) que é definido como o valor médio de ingestão de energia proveniente da alimentação necessária para a manutenção do balanço energético de indivíduos saudáveis (Fisberg, Marchioni \& Slater Villar, 2005), devendo-se utilizar no cálculo o coeficiente de atividade física, que corresponde ao grau de atividade física (em inglês: Physical Activity Level - PAL) do indivíduo. Outra equação conhecida na prática clínica é a proposta por Schofield (1985), que estima o gasto energético basal (GEB) (em inglês: Basal Energy Expenditure). O termo basal é usado para diferenciar a energia gasta durante a realização de atividades físicas e em estado de repouso (Henry, 2005).

Uma vez que, alterações no consumo alimentar e no gasto energético resultam do desequilíbrio ou não da ingestão calórica e atividade física, e frente à complexidade que permeia a avaliação do consumo alimentar de grupos populacionais 
(especialmente os adolescentes que estão mais vulneráveis a pressões sociais diversas), através de explicações sistemáticas e discussão intersubjetiva (Köche, 2011), pressupõe-se que os adolescentes estão expostos a comportamentos de risco, como pular refeições, sedentarismo e alimentar-se fora de casa, e que o consumo alimentar e as necessidades nutricionais são incompatíveis. Assim, o presente estudo tem o propósito de contribuir com uma abordagem metodológica ao comparar as necessidades energéticas, obtidas por meio de equações preditivas, com os dados de consumo alimentar de uma população de adolescentes escolares, de municípios baianos. Além disso, buscou-se conhecer o espaço alimentar e as refeições que são realizadas por essa população, na tentativa de entender as vulnerabilidades que envolvem essa comensalidade (o quê, quando e onde comer?).

\section{Metodologia}

Trata-se de um estudo transversal, aninhado ao ensaio comunitário randomizado controlado do projeto de pesquisa “Desenvolvimento e avaliação de uma intervenção intersetorial no âmbito da Estratégia da Saúde da Família (ESF) sobre padrões alimentares e de atividade física de adolescentes" (nominado PROSE - promovendo saúde na escola), que foi realizado com adolescentes entre 12 a 18 anos e 11 meses de escolas públicas do ensino médio, nos anos de 2013 e 2014.

Figura 1. Fluxograma do Projeto PROSE realizado com adolescentes escolares da rede pública estadual de dez municípios baianos. Projeto PROSE, Bahia/2013-2014.

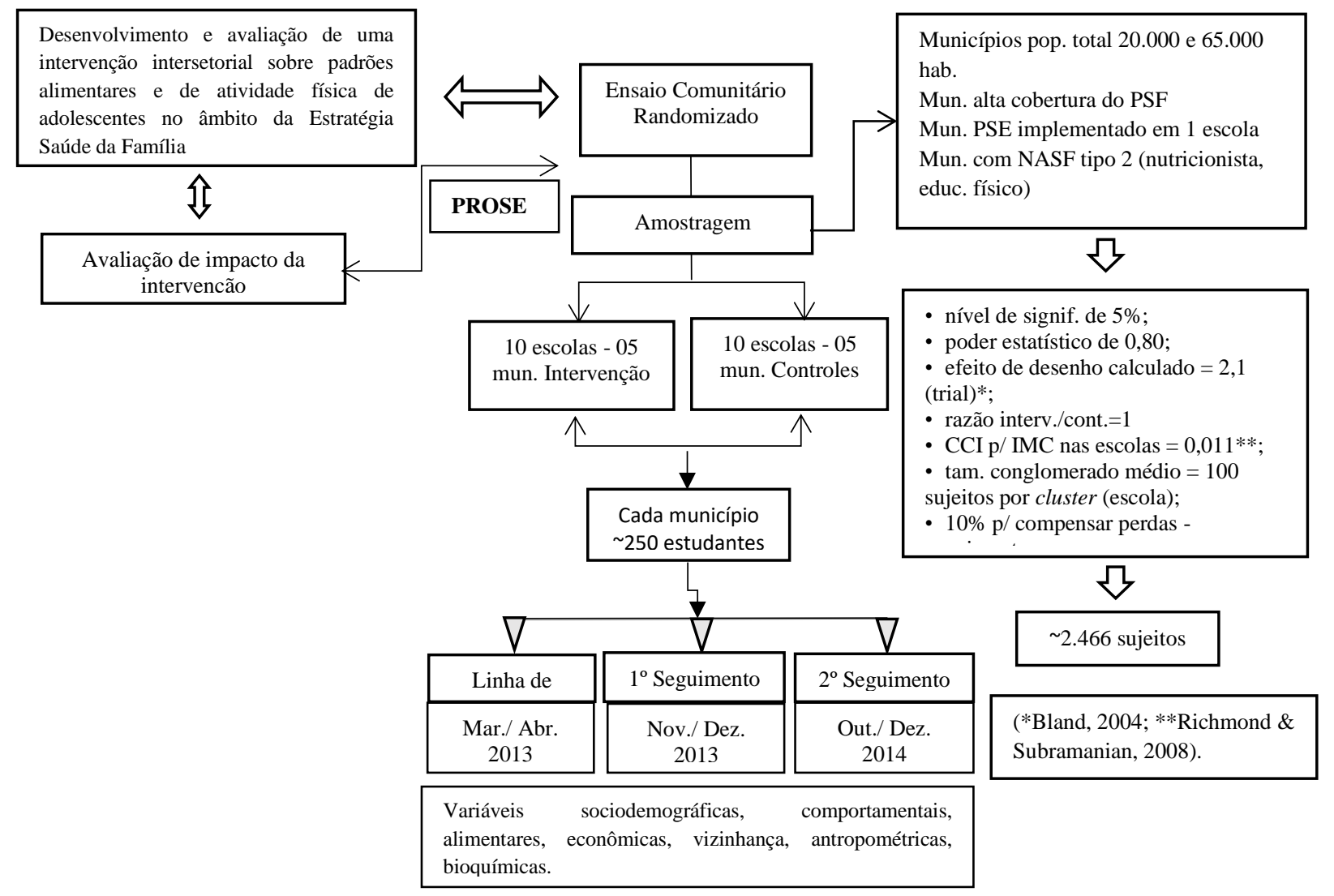

Fonte: Extraído de Fonseca (2017).

A unidade de análise do projeto PROSE eram os adolescentes escolares, residentes de municípios baianos que atenderam aos critérios de inclusão (Figura 1). Após aplicação de todos os critérios restaram dez municípios, que foram pareados, e alocados de forma aleatória, no grupo controle e no grupo intervenção. Em cada município selecionado, controle ou intervenção, todas as escolas de ensino médio localizadas na área urbana e com pelo menos 100 alunos, foram incluídas na pesquisa e, todos os 
estudantes das escolas selecionadas e matriculados no primeiro e segundo ano do ensino médio foram elegíveis para participar do estudo realizado no período de avaliação, totalizando vinte escolas em dez municípios (Fonseca-Barroso, Aquino \& Amorim, 2021).

\section{População do estudo}

Por se tratar de municípios do interior baiano e considerando a logística de campo durante a fase de implementação do PROSE, para este estudo limitou-se o número de escolas participantes, uma em cada município. Foram convidados a participar deste estudo, todos os adolescentes integrantes do PROSE de cada escola selecionada, ou seja, a primeira escola visitada no município. Os adolescentes escolares entre 12 a 18 anos e 11 meses, matriculados no primeiro ou segundo ano do ensino médio, de ambos os sexos, que aceitaram participar do estudo e que responderam ao QFA e a três RA foram incluídos. Iniciou-se com 113 adolescentes que responderam ao QFA e os RA. Indivíduos que não completaram os três registros alimentares ou o QFA e ainda, indivíduos com implausibilidade de consumo (<500 kcal ou >7000 kcal) foram excluídos das análises, restando em 85 indivíduos.

\section{Coleta de dados}

No baseline do PROSE foi aplicado o QFA quantitativo, composto por 146 itens alimentares, estipulando-se os seis meses precedentes à sua aplicação como unidade de tempo para estimar a frequência de consumo de alimentos, de modo a minimizar possíveis vieses de informação devido a dificuldades dos adolescentes em relembrar seu consumo alimentar. O QFA utilizado foi validado para a população do estudo (Fonseca-Barroso, Aquino \& Amorim, 2021).

Após a aplicação dos inquéritos do PROSE, os adolescentes foram convidados a preencher três registros alimentares, que tem como vantagem a não dependência da memória do participante do estudo (Willett, 2013), vez que registra o consumo alimentar à medida que ocorre. Para isso, os adolescentes receberam orientações sobre o preenchimento, em relação às porções, horários e dias, preferencialmente dois dias da semana alternados e um dia do final de semana. Junto com o RA foi entregue um álbum contendo desenhos de utensílios e volume, para facilitar a identificação das medidas caseiras e minimizar o viés de memória. E de forma a evitar que a coleta de dados por um método influenciasse as respostas do outro, os registros somente foram entregues aos adolescentes após a aplicação do método teste - QFA (Cade, Thompson, Burley \& Warm, 2002).

A gramatura das porções alimentares foi estabelecida conforme descrito por Fonseca-Barroso, Aquino \& Amorim, (2021). Para porções de preparações ou alimentos referidos nos RA que se diferenciaram daqueles contido no material de apoio distribuído pela equipe de campo, foram utilizadas as informações das bases de dados referidas além de receitas padronizadas e, em informações dos rótulos de alimentos industrializados consultadas em supermercados e na internet. Seguido da conversão para unidades de peso e volume e o cálculo do valor nutricional diário dos alimentos.

E com o intuito de se conhecer os hábitos de realização das refeições pelos adolescentes, estimou-se a partir dos RA, a frequência de cada refeição nos últimos seis meses, além do local em que as realizavam, ou seja, considerando-se três dias de RA por participante, e uma diversidade de até sete refeições ao dia (café da manhã, lanches (manhã, tarde, noite e extra), almoço e jantar) o quantitativo máximo esperado foi de 21 refeições por adolescente.

O Questionário Internacional de Atividade Física (International Physical Activity Questionnaire - IPAQ) foi aplicado a todos os participantes do Projeto PROSE. O IPAQ estima o tempo semanal gasto em atividades físicas e computa os equivalentes metabólicos (em inglês, metabolic equivalents - METs). METs são múltiplos da taxa metabólica de repouso e um MET - minuto é obtido multiplicando-se o MET escore de uma atividade pelos minutos realizados. METs - minutos são equivalentes a quilocalorias (kcal) para um indivíduo de 60 quilogramas $(\mathrm{kg})$, e podem ser calculados usando a seguinte equação: MET - min. x (peso (kg) / $60 \mathrm{~kg}$ ). MET - min./dia ou MET - min./semana podem ser computados, embora o último seja usado 
com mais frequência (IPAQ Research Committee et al., 2005), distribuídas em quatro dimensões (trabalho, transporte, tarefas domésticas e lazer) e do tempo despendido por semana na posição sentada (Benedetti, Antunes, Rodriguez- Añez, Mazo \& Petroski, 2007).

As medidas antropométricas foram coletadas em duplicata por dois avaliadores independentes, profissionais de nível superior - nutricionistas, enfermeiros ou graduandos em Saúde Coletiva, em ambiente reservado em cada escola e de forma padronizada (Lohman, Roche \& Martorell, 1988). Peso e altura foram obtidos utilizando-se, respectivamente, balança eletrônica portátil Marte ${ }^{\circ}$ e estadiômetro portátil, marca Leicester Height Measure ${ }^{\circledR}$, admitindo-se variação mínima de $100 \mathrm{~g}$ e 0,5cm, para ambas as medidas. A circunferência da cintura (CC) foi aferida com fita métrica flexível e inelástica (TBWß, São Paulo, Brasil). A CC foi medida no ponto médio entre a crista ilíaca e a face externa da última costela, aceitando-se variações de 0,5 cm entre as duas medidas, adotando-se a média entre os valores (Freedman, Serdula, Srinivasan, \& Berenson, 1999).

\section{Processamento e análise dos dados}

Para estimar as necessidades energéticas dos adolescentes escolares, utilizaram-se as equações preditivas das DRI's (Institute of Medicine, 2002/2005) e de Schofield (1985) (Quadro 1). Para o cálculo do Gasto energético total (GET) ou Valor energético total (VET) utilizou-se a equação GET = GEB * fator atividade física (PAL), onde GEB = gasto energético basal, $\mathrm{PAL}=$ physical activity level.

Quadro 1. Equações para o cálculo da necessidade média estimada de energia de adolescentes, considerando sexo e idade.

\begin{tabular}{|c|c|c|c|}
\hline \multicolumn{4}{|c|}{ IOM (2002/2005) } \\
\hline Estágio da Vida/ Sexo & EER (kcal) & PAL & DP (kcal) \\
\hline $\begin{array}{l}\text { Adolescentes do sexo } \\
\text { Masculino } \\
9-18 \text { anos (eutróficos) }\end{array}$ & $\begin{array}{l}88,5-61,9 \times \text { Idade }(\text { anos })+[\text { NAF x }(26,7 x \\
\text { Peso }(\mathrm{kg})+903 \text { x Estatura }(\mathrm{m}))]+25(\mathrm{kcal})\end{array}$ & $\begin{array}{l}\text { 1,00 Sedentário; } 1,13 \text { Pouco ativo; } \\
\text { 1,26 Ativo; } 1,42 \text { Muito ativo }\end{array}$ & 58 \\
\hline $\begin{array}{l}\text { Adolescentes do sexo Feminino } \\
9-18 \text { anos (eutróficos) }\end{array}$ & $\begin{array}{l}135,3-30,8 \times \text { Idade }(\text { anos })+[\text { NAF x }(10 \mathrm{x} \\
\text { Peso }(\mathrm{kg})+934 \mathrm{x} \text { Estatura }(\mathrm{m}))]+25(\mathrm{kcal})\end{array}$ & $\begin{array}{l}\text { 1,00 Sedentário; } 1,16 \text { Pouco ativo; } \\
\text { 1,31 Ativo; } 1,56 \text { Muito ativo }\end{array}$ & 68 \\
\hline \multicolumn{4}{|c|}{ Equação de Schofield (1985) } \\
\hline Estágio da Vida/Sexo & GEB (kcal) & \multicolumn{2}{|l|}{ PAL } \\
\hline $\begin{array}{l}\text { Adolescentes do sexo } \\
\text { Masculino } \\
10-18 \text { anos }\end{array}$ & $16,25(\mathrm{P})+1,372(\mathrm{E})+515,5$ & \multicolumn{2}{|c|}{$\begin{array}{l}\text { 1,00 Sedentário; } 1,13 \text { Pouco ativo; 1,26 Ativo; 1,42 } \\
\text { Muito ativo }\end{array}$} \\
\hline $\begin{array}{l}\text { Adolescentes do sexo Feminino } \\
\qquad 10-18 \text { anos }\end{array}$ & $8,365(\mathrm{P})+4,65(\mathrm{E})+200$ & \multicolumn{2}{|c|}{$\begin{array}{l}\text { 1,00 Sedentário; } 1,16 \text { Pouco ativo; 1,31 Ativo; 1,56 } \\
\text { Muito ativo }\end{array}$} \\
\hline
\end{tabular}

Nota: EER: Estimated Energy Requeriment (Necessidade Estimada de Energia = Fator por Idade e Sexo, dados de Idade, Peso Corporal (Atual) e Altura, devem ser inseridos nas equações em anos, quilogramas $(\mathrm{Kg})$ e metros $(\mathrm{M})$, respectivamente, e acrescidos de $25 \mathrm{Kcal}$ de energia para deposição de tecidos); GEB = P - peso real (Kg), E: estatura real (Cm); PAL: Physical Activity Level (Coeficientes De Atividade Física); GEB = Gasto Energético Basal em Kcal; PAL = Physical Activity Level . Fonte: Adaptado de Institute of Medicine (2002-2005) e de Schofield, (1985).

Baseado no gasto energético obtido no IPAQ, os participantes do estudo foram classificados de acordo com a intensidade de atividade física, considerando a conversão em METs, adotando-se os seguintes valores para cada intensidade: Caminhada - 3,3 METs/sem; Moderada - 4,0 METs/sem e Vigorosa - 8,0 Mets/sem. Em seguida, classificaram-se os adolescentes em quatro categorias, com base no estudo de Cruciani et al. (2009): a) Sedentário - <600 METs/sem; b) Pouco 
ativo - 600 - 1500 METs/sem; c) Ativo - > 1500 a < 3000 METs/sem; d) Muito ativo - > 3000 METs/sem (IPAQ Research Committee et al., 2005). Em seguida, a variável foi dicotomizada considerando todos os domínios de tipos de atividade física em abaixo ou acima/igual a 300 minutos por semana.

O estado antropométrico foi classificado pelo índice corporal (IMC) (peso $(\mathrm{kg}) / \mathrm{estatura}\left(\mathrm{m}^{2}\right)$ ), e adotados os percentis segundo sexo e idade, categorizados em: magreza (< percentil 3), eutrofia (> percentil 3 e < percentil 85), sobrepeso (> percentil 85 e < percentil 97) e obesidade (> 97) (World Health Organization, 2007a; 2007b). Os adolescentes com sobrepeso (> percentil 85) ou obesidade (< percentil 85) também foram classificados como “excesso de peso". A circunferência da cintura foi considerada aumentada quando a medida foi maior ou igual ao percentil 90 para idade e sexo da própria amostra considerando estudos prévios (International Diabetes Federation, 2007; Pereira et al., 2011), e categorizada em elevada (> percentil 90) ou adequada. A razão circunferência/estatura (RCE) permite o estabelecimento de um ponto de corte único e aplicável à população geral, independente do sexo, idade e etnia, e foi dicotomizada em abaixo ou acima de 0,50 - ponto de corte para diagnóstico do excesso de gordura abdominal (Ashwell \& Hsieh, 2005; Pereira et al., 2011).

Os dados foram analisados de forma descritiva utilizando-se frequência, simples e absoluta, para as variáveis categóricas, e a média e respectivos desvios padrão (DP) para as contínuas, sendo testada a normalidade por meio do Teste de Shapiro Wilk. Foi realizada análise bivariada e aplicado o teste do Qui-quadrado de Pearson e teste exato de Fischer, para comparar as estimativas do consumo alimentar, e o nível de atividade física, por sexo. A adequação percentual, entre as necessidades energéticas estimadas (GEB, GET e EER) e o consumo médio de energia obtidos no QFA e RA, foi calculada com base na EER +2DP para a faixa etária entre 03 a 18 anos (DP sexo mas.: 58; DP sexo fem.: 68). Foi aplicado o teste de hipótese de diferenças das médias, entre os métodos preditivos das necessidades energéticas (Institute of Medicine, 2002/2005; Schofield, 1985) e o consumo energético (RA e QFA), via o ajuste de modelo linear misto sem covariáveis, tendo como desfecho as diferenças entre os dois métodos (a distribuição de normalidade das diferenças foram testadas por meio do teste de Shapiro Wilk). Assim, a não rejeição da hipótese nula de que o intercepto é zero (Ho: $\beta o=0)$ indica proximidade do consumo alimentar médio (QFA ou RA) com as necessidades energéticas dos adolescentes. Adotou-se nível de significância de 5\% para todas as análises. Para todas as análises considerou-se o efeito do desenho do PROSE, em conglomerados e, utilizou-se o software STATA (Stata Corporation, EEUU) versão 15.0.

\section{Aspectos éticos}

O projeto de pesquisa foi aprovado pelo Comitê de Ética do Instituto de Saúde Coletiva da Universidade Federal da Bahia (CCAE 09709112.3.0000.5030) pelo parecer 182.685, de 4 de janeiro de 2013. Em conformidade com a Resolução n466/2012 do Conselho Nacional de Saúde, assegurou-se o anonimato dos participantes, sigilo e confidencialidade das informações coletadas.

\section{Resultados}

A população do estudo foi composta por 85 adolescentes, com idade entre 13 a 18 anos, média de 15,7 anos de idade, do sexo feminino $(78,8 \%)$, autodeclarados pretos ou pardos $(51,8 \%)$ e estudavam no turno vespertino $(63,9 \%)$. Em relação à família, verificou-se que os chefes da família trabalhavam $(68,8 \%)$, e encontrou-se elevada prevalência de responsáveis pela família que não cursaram o ensino médio (44,3\%). A maioria dos escolares considerava sua situação de saúde boa ou regular $(61,2 \%)$ e praticavam mais de 300 minutos de atividade física por semana $(69,5 \%)$ incluindo todos os domínios e tipos de atividades (lazer, doméstico, transporte e trabalho) com 61\% classificado como ativo ou muito ativo. Em contraponto, a frequência de comportamento sedentário, a exemplo de tempo de tela, por mais de duas (02) horas ao dia também foi elevada 
(88,0\%), ou seja, em média 4,7 horas (DP: 3,5) eram despendidas em atividades sedentárias. Os dados de circunferência de cintura e razão cintura/estatura permitiram identificar uma população de adolescentes na faixa desejada para a idade e idade/altura $(92,9 \%)$ (Tabela 1).

Tabela 1. Características sociodemográficas, comportamentais e antropométricas de adolescentes escolares da rede pública estadual de municípios baianos. Projeto PROSE, Bahia/2013.

\begin{tabular}{|c|c|c|c|}
\hline Características & Categorias & $\mathbf{n}$ & $\%$ \\
\hline \multirow{2}{*}{$\operatorname{Sexo}(n=85)$} & Feminino & 67 & 78,8 \\
\hline & Masculino & 18 & 21,2 \\
\hline \multirow{5}{*}{$\operatorname{Raça}(n=85)$} & Branco & 6 & 7,1 \\
\hline & Preto/pardo & 44 & 51,8 \\
\hline & Indígena/amarelo & 9 & 10,6 \\
\hline & Não se enquadra & 2 & 2,3 \\
\hline & Não responderam & 24 & 28,2 \\
\hline \multirow{2}{*}{ Turno escolar $(n=61)$} & Matutino & 22 & 36,1 \\
\hline & Vespertino & 39 & 63,9 \\
\hline \multirow{2}{*}{$\begin{array}{l}\text { Chefe da família trabalha? (n } \\
=61)\end{array}$} & Sim & 42 & 68,8 \\
\hline & Não & 19 & 31,2 \\
\hline \multirow{2}{*}{$\begin{array}{l}\text { Escolaridade do chefe da família } \\
(n=61)\end{array}$} & $<8$ anos de estudo & 27 & 44,3 \\
\hline & $\geq 8$ anos de estudo & 34 & 55,7 \\
\hline \multirow{2}{*}{ Considera sua saúde $(\mathbf{n}=\mathbf{8 5}$ ) } & Boa/muito boa & 52 & 61,2 \\
\hline & Regular/ruim & 33 & 38,8 \\
\hline \multirow{2}{*}{ Atividade Física (IPAQ) (n=82) } & $<300 \mathrm{~min} / \mathrm{semana}$ & 25 & 30,5 \\
\hline & $\geq 300 \mathrm{~min} / \mathrm{semana}$ & 57 & 69,5 \\
\hline \multirow{2}{*}{$\begin{array}{l}\text { Comportamento sedentário }(n= \\
\text { 83) }\end{array}$} & $<02$ horas/dia & 10 & 12,0 \\
\hline & $\geq 02$ horas $/$ dia & 73 & 88,0 \\
\hline \multirow{2}{*}{ Circunferência da cintura $(\mathrm{n}=85)$} & $<80,15$ & 80 & 94,1 \\
\hline & $\geq 80,15$ & 5 & 5,9 \\
\hline \multirow{2}{*}{ Razão Cintura/estatura (n=85) } & $<0,5$ & 79 & 92,9 \\
\hline & $\geq 0,5$ & 6 & 7,1 \\
\hline \multirow{4}{*}{ Atividade Física (IPAQ) (n=82) } & Sedentário $-<600$ METs/sem & 10 & 12,2 \\
\hline & Pouco ativo - 600 a $1500 \mathrm{METs} / \mathrm{sem}$ & 22 & 26,8 \\
\hline & Ativo $->1500 \mathrm{a}<3000 \mathrm{METs} / \mathrm{sem}$ & 22 & 26,8 \\
\hline & Muito ativo $->3000 \mathrm{METs} / \mathrm{sem}$ & 28 & 34,2 \\
\hline
\end{tabular}

Fonte: Autores, Salvador- Bahia (2021).

Com base nos dados obtidos nos registros alimentares foi possível identificar o tipo de refeição e o local onde eram realizadas. Observou-se que as principais refeições do dia, café da manhã e almoço foram as mais frequentes, seguidas pelo jantar, com percentual de realização de tais refeições variando entre $89 \%$ a aproximadamente $95 \%$. E o domicílio do estudante foi o mais utilizado para a realização de todas as refeições, ainda que as chamadas pequenas refeições, em especial os lanches da manhã e tarde, tenham sido realizados tanto na escola (26\% - lanche da tarde) quanto em ambientes diversos (16,2\% - lanche da manhã) a exemplo de casa de familiares, lanchonetes, entre outros (Tabela 2). 
Tabela 2. Distribuição das refeições por local de realização das refeições de adolescentes escolares da rede pública estadual de municípios baianos. Projeto PROSE, Bahia/2013.

\begin{tabular}{|c|c|c|c|c|c|c|c|}
\hline Local/Refeição & $\begin{array}{c}\text { Café da } \\
\text { manhã } \\
\text { n (\%) }\end{array}$ & $\begin{array}{c}\text { Lanche da } \\
\text { manhã } \\
\text { n }(\%)\end{array}$ & $\begin{array}{l}\text { Almoço } \\
\text { n (\%) }\end{array}$ & $\begin{array}{c}\text { Lanche da } \\
\text { tarde } \\
\text { n }(\%)\end{array}$ & $\begin{array}{l}\text { Jantar } \\
\text { n }(\%)\end{array}$ & $\begin{array}{c}\text { Lanche da } \\
\text { noite } \\
\text { n }(\%) \\
\end{array}$ & $\begin{array}{c}\text { Lanche } \\
\text { extra } \\
\text { n }(\%)\end{array}$ \\
\hline Casa & $223(93,7)$ & $87(58,8)$ & $215(89,6)$ & $115(56,4)$ & $184(82,1)$ & $98(79,7)$ & $39(72,2)$ \\
\hline $\begin{array}{l}\text { Fora de casa (locais } \\
\text { diversos)! }\end{array}$ & $9(3,8)$ & $24(16,2)$ & $17(7,1)$ & $29(14,2)$ & $21(9,4)$ & $17(13,8)$ & $6(11,1)$ \\
\hline Não informado & $9(3,8)$ & $9(6,1)$ & $9(3,8)$ & $10(4,9)$ & $21(9,4)$ & $10(8,1)$ & $9(16,7)$ \\
\hline Escola & $1(0,4)$ & $29(19,6)$ & $1(0,4)$ & $53(26,0)$ & $1(0,4)$ & $0(0)$ & $3(5,6)$ \\
\hline Trabalho & $0(0)$ & $2(1,4)$ & $0(0)$ & $0(0)$ & $0(0)$ & $0(0)$ & $0(0)$ \\
\hline $\begin{array}{l}\text { Total } \\
\text { \% refeição realizadas* }\end{array}$ & $\begin{array}{c}242(100) \\
94,9\end{array}$ & $151(100)$ & $\begin{array}{c}242(100) \\
94,9\end{array}$ & 207 (100) & $\begin{array}{c}227(100) \\
89,0\end{array}$ & $125(100)$ & $57(100)$ \\
\hline
\end{tabular}

! Locais diversos: casa de parentes e amigos, lanchonetes, restaurantes, quiosques. *Percentual de realização das principais refeições: considerando três dias de registro alimentar (com expectativa de no mínimo três principais refeições (03) ao dia) e o número amostral de 85, o n esperado seria de 255 para cada refeição principal (café da manhã, almoço e jantar). Fonte: elaborado pelas autoras, Salvador- Bahia, 2019.

$\mathrm{Na}$ análise bivariada, verificou-se que tanto as meninas quanto os meninos apresentavam-se eutróficos em sua maioria $(83,1 \%)$ e não se encontrou diferença estatisticamente significativa entre sexo e estado antropométrico da população (pvalor >0,05). Quanto à intensidade da atividade física, observou-se que as adolescentes apresentaram maior prevalência no nível ativo, enquanto os meninos nível muito ativo (dados não mostrados), porém não se encontrou diferenças estatisticamente significantes ( $\mathrm{p}$-valor $>0,05$ ) entre o tempo gasto em todos os domínios de atividade física (em minutos) e o sexo dos adolescentes (Tabela 3).

Tabela 3. Distribuição do estado antropométrico e nível de atividade física por sexo dos adolescentes escolares da rede pública estadual de municípios baianos. Projeto PROSE, Bahia/2013.

\begin{tabular}{lcccccc}
\hline \multirow{2}{*}{ Variáveis } & \multicolumn{2}{c}{ Feminino } & \multicolumn{2}{c}{ Masculino } & \multicolumn{2}{c}{ Total } \\
\cline { 2 - 7 } & $\mathrm{n}$ & $\%$ & $\mathrm{n}$ & $\%$ & $\mathrm{n}$ & $\%$ \\
\hline Estado antropométrico* & & & & & & \\
Baixo Peso & 02 & 3,0 & 03 & 17,7 & 05 & 6,0 \\
Eutrofia & 56 & 84,9 & 13 & 76,4 & 69 & 83,1 \\
Excesso de peso & 08 & 12,1 & 01 & 5,9 & 09 & 10,9 \\
Atividade Física** & & & & & & \\
$<300 \mathrm{~min} /$ semana & 19 & 29,7 & 6 & 33,3 & 25 & 30,5 \\
$\geq 300 \mathrm{~min} / \mathrm{semana}$ & 45 & 70,3 & 12 & 66,7 & 57 & 69,5 \\
\hline
\end{tabular}

*p-valor do Teste Exato de Fischer: 0,12 (Estado Antropométrico); ** p-valor do Qui-Quadrado de Pearson - 0,58 (Atividade Física). Fonte: Autores, Salvador- Bahia (2019).

Conforme esperado, as necessidades energéticas dos meninos se mostraram, em média, maior que as das meninas (>20\% - GEB; >14,6\% - GET; e >37,3\% - EER), resultado similar foi encontrado para o consumo alimentar (>22,6\% e >33,6\% no RA e QFA, respectivamente) além, de uma diferença de aproximadamente $36 \%$ a mais no dispêndio energético médio (Tabela 4). 
Tabela 4. Médias das necessidades energéticas e do consumo alimentar obtido no RA e QFA de adolescentes escolares da rede pública estadual de municípios baianos. Projeto PROSE, Bahia/2013.

\begin{tabular}{llcccc}
\hline \multirow{2}{*}{ Categoria } & \multirow{2}{*}{ Variáveis } & Média (EP) & IC95\% & Média (EP) & Masculino \\
\cline { 3 - 6 } Necessidades & GEB & $1390,83(7,2)$ & $1374,6-1407,0$ & $1662,06(38,7)$ & $1570,4-1753,7$ \\
energéticas (Kcal) & GET & $1822,53(44,4)$ & $1722,1-1923,0$ & $2088,5(45,1)$ & $1981,9-2195,1$ \\
& EER & $1869,31(21,7)$ & $1820,2-1918,4$ & $2567,26(80,1)$ & $2377,8-2756,7$ \\
Dispêndio energético & MET’s & $2717,10(350,6)$ & $1923,9-3510,3$ & $3691,82(683.3)$ & $2146,2-5237,5$ \\
& & & & & \\
Consumo alimentar & RA & $2183,2(88,2)$ & $1983,8-2382,7$ & $2675,78(118,1)$ & $2403,4-2948,2$ \\
$($ Kcal) & QFA & $3319,9(132,9)$ & $3019,3-3620,5$ & $4437,17(292,7)$ & $3762,3-5112,0$ \\
\hline
\end{tabular}

EP: erro padrão; EER: Estimated Energy Requeriment; GEB: Basal Energy Expenditure; GET: Total Energy Expenditure; METs: metabolic equivalents, RA: Registro Alimentar; QFA: Questionário de Frequência Alimentar. Fonte: Autores, Salvador- Bahia (2019).

Verificou-se um consumo alimentar caloricamente inadequado quando se compara à recomendação energética através do EER. Observou-se que tanto o RA $(83,75 \%)$ quanto o QFA $(96,25 \%)$ apresentaram alto percentual de inadequação (Tabela $5)$.

Tabela 5. Adequação das necessidades energéticas preditivas frente ao consumo alimentar (obtido no RA e QFA) e teste de hipótese das diferenças de médias entre os métodos, aplicados a adolescentes escolares da rede pública estadual de municípios baianos. Projeto PROSE, Bahia/2013.

\begin{tabular}{|c|c|c|c|c|c|c|}
\hline \multirow{2}{*}{ Variáveis } & \multicolumn{2}{|c|}{ Feminino $(n=63)$} & \multicolumn{3}{|c|}{ Masculino (n=17) } & \multirow[t]{2}{*}{ P-valor* } \\
\hline & $\begin{array}{c}\text { Adequado } \\
\text { n (\%) }\end{array}$ & $\begin{array}{c}\text { Inadequado } \\
\text { n (\%) }\end{array}$ & P-valor* & $\begin{array}{c}\text { Adequado } \\
\text { n (\%) }\end{array}$ & $\begin{array}{c}\text { Inadequado } \\
\mathrm{n}(\%)\end{array}$ & \\
\hline RA & $14(22,2)$ & $49(77,8)$ & 0,01 & $0(0)$ & $17(100)$ & 0,53 \\
\hline QFA & $3(4,8)$ & $60(95,2)$ & 0,00 & $2(11,8)$ & $15(88,2)$ & 0,00 \\
\hline
\end{tabular}

*Cômputo da adequação da EER - DRI'S (Institute of Medicine, 2002-2005) considerando + 2DP (específicos para sexo e idade). *P-valor do teste de hipótese de diferenças das médias entre os métodos, via modelo linear misto sem covariáveis $(\mathrm{H0}$ : EER - $(\mathrm{QFA} / \mathrm{RA})=0)$. Fonte: Autores, Salvador- Bahia (2019).

Os resultados do teste de hipóteses para comparação das diferenças médias das necessidades energéticas - preditas por equações e do consumo energético obtido por dois instrumentos de avaliação do consumo alimentar - mostraram que as diferenças médias foram estatisticamente significantes para as adolescentes, independente do instrumento de consumo utilizado ( $\mathrm{p}$-valor <0,05), porém, entre os meninos as diferenças médias não foram estatisticamente significantes (p-valor >0,05) quando avaliadas por meio do RA (Tabela 5). Como teste de sensibilidade aplicou-se o teste de Wald para avaliar a hipótese nula de que as diferenças entre os valores energéticos preditos (GET e EER) e o consumo alimentar (QFA e RA) eram zero, de acordo com o sexo dos adolescentes, sendo rejeitada a hipótese nula, e confirmando as diferenças entre a informação do consumo alimentar e as necessidades energéticas, mesmo para os meninos (p-valor $<0,05$ ). 


\section{Discussão}

Este estudo permitiu comparar características relacionadas à manutenção do balanço energético de adolescentes escolares saudáveis e o consumo alimentar obtido em dois diferentes instrumentos, sendo verificado elevados percentuais de inadequação da necessidade e do consumo, independente do sexo, com superestimação do consumo alimentar obtido no RA e QFA.

Diferentemente do encontrado em outros estudos (Araújo, Toral, Silva, Velásquez-Melendez \& Dias, 2009; Lima et al., 2017; Mendonça, Carvalho, Oliveira, Assis \& Moreira, 2019), a população adolescente deste estudo apresentou uma baixa prevalência de excesso de peso (sobrepeso - 8,5\% e obesidade - 2,4\%). Corroborando esse dado, têm-se uma elevada prevalência de dados antropométricos adequados (IMC, CC e RCE), com elevado nível de atividade física. Porém o comportamento sedentário estava presente de forma elevada (88\%). Costa et al. (2018) ao analisarem o comportamento sedentário entre adolescentes brasileiros, detectaram que 68,1\% (IC95\%: 67,7-68,7) dos escolares referiram passar mais de duas horas por dia desempenhando atividades sentados. E De Souza, Marques e Reuter (2020), em estudo realizado com 795 crianças e adolescentes, observaram que 57,1\% gastavam a mesma quantidade de horas diárias em frente à tela da TV, computador ou videogame.

Foi possível identificar que o espaço de comensalidade da população do estudo era o próprio domicílio, na qual eram realizadas aproximadamente $80 \%$ ou mais das grandes refeições, fato que pode ser atribuído à fatores como tratar-se de uma população oriunda de cidades de médio porte, do interior baiano com população entre 20.000 e 60.000 habitantes, onde se resguarda modos de comer, relações de comensalidade e identidade alimentar da população mas que não os deixam imunes a vida contemporânea onde são apresentadas constantemente mudanças que podem atingir também as famílias rurais, fazendo com que busquem formas de se adaptar, criando alternativas para lidar com a nova realidade (De Souza lima, Neto \& Farias, 2015). Discrepâncias entre as zonas urbana e rural podem ser encontradas na Pesquisa de Orçamentos Familiares 2017-2018 (Instituto Brasileiro de Geografia e Estatística, 2020) quanto à despesa per capita mensal com alimentação no Brasil.

Residentes da área urbana somaram $89,1 \%$ das despesas per capita encontradas no Brasil e os da zona rural somente 10,9\%. Na região Nordeste, onde se desenvolveu este estudo, verificou-se que as despesas per capita somaram R $\$ 147,45$, mantendo-se as discrepâncias entre as zonas urbana e rural (87,8\% e 12,2\%, respectivamente). Outro marcador das diferenças encontradas na região nordeste é a despesa com alimentação fora do domicílio, ficando em torno de $8 \%$ na zona rural quando comparadas com as despesas totais fora do domicílio (Instituto Brasileiro de Geografia e Estatística, 2020), caracterizando uma desigualdade no consumo e que respondem aos dados encontrados. Outros estudos também evidenciaram diferenças nos padrões alimentares de adolescentes que residem em capitais ou cidades menores ou em área urbana ou rural (Aranceta, Perez-Rodrigo, Ribas \& Serra-Majem, 2003; Bezerra \& Sichieri, 2010). A renda familiar da população do estudo não foi avaliada, entretanto, a escolaridade do chefe da família $(44,3 \%$ com < 08 anos de estudo) e o fato se se tratar de estudantes de escolas públicas podem ser tomados como proxy das condições socioeconômicas e de acordo com Rodrigues et al., (2012), condições socioeconômicas desfavoráveis podem ser associadas a padrões alimentares tradicionais, os quais são reconhecidos como protetores para a saúde.

Tais características, como realizar no mínimo 3 refeições, em companhia da família, podem auxiliar na construção de barreiras que minimizem as vulnerabilidades que envolvem a adolescência, em especial os que estudam em escolas públicas. O que comer, quando e onde são questões da comensalidade que impactam nesse importante determinante social da saúde, que é a alimentação. No estudo de Simões, Machado \& Höfelmann, (2021), com uma amostra de 1.232 escolares, verificou-se que mais da metade realizava as refeições do almoço $(58,4 \%)$ ou jantar $(61,9 \%)$ com a família diariamente. Outro estudo (Martins et al., 2019), encontrou o hábito de fazer as refeições com os pais, pelo menos 5 dias da semana, em $74 \%$ dos adolescentes, sendo esta prática mais frequente entre os meninos, os mais novos, filhos de mulheres com menor escolaridade, que estudavam em escolas 
públicas e não residiam em capitais. Esses autores ainda encontraram uma associação protetora entre a frequência de realizar as refeições com os pais e o consumo frequente dos alimentos marcadores de alimentação saudável (consumo de frutas $(\mathrm{RP}=1,34$; IC95\%: 1,28-1,39) e hortaliças (RP = 1,34; IC95\%: 1,28-1,39)), e negativamente associado com o consumo de alimentos não saudáveis a exemplo de guloseimas, ultraprocessados salgados e salgados fritos (Martins et al., 2019). Resultados que demonstram a importância do comportamento encontrado neste estudo.

Não se evidenciou o hábito de "pular" o café da manhã na população do estudo. Ao contrário, aproximadamente $95 \%$ da população relatou a realização dessa importante refeição, diferindo do estudo de Simões, Machado \& Höfelmann, (2021) que encontraram um consumo regular do café da manhã de 58,6\% (IC95\% 53,9; 63,2), e 17,7\% não realizavam esta refeição em nenhum dia da semana. O café da manhã melhora os aspectos cognitivos, como desempenho acadêmico, concentração e memória (Trancoso, Cavalli \& Proença, 2010). Há evidências de que os jovens que relatam realizar essa refeição regularmente têm melhor perfil nutricional comparados aos que a omitem, esses comportamentos alimentares, associados ao sedentarismo, são fatores contribuintes para o aumento contínuo da prevalência de obesidade e outras DCNTs (Trancoso, Cavalli \& Proença, 2010; Araki et al., 2011), proteção que foi encontrada neste estudo haja vista a prevalência de excesso de peso da população (10,9\%).

A alimentação escolar é de suma importância, podendo ser considerada a principal refeição do dia para muitos estudantes proveniente da rede pública, especialmente quando se observa o estudo de Valentim, et al., (2017) que evidenciou que a adesão à alimentação escolar foi 32\% maior entre os adolescentes mais vulneráveis economicamente (renda domiciliar per capita igual ou inferior a um salário-mínimo). Esses autores encontraram uma prevalência de adesão à alimentação escolar de $57,7 \%$, porém, apenas 19,8\% caracterizavam-se como adesão efetiva, esses dados e os nossos (somente os lanches eram realizados na escola) podem ser justificados pelo fato de que a alimentação escolar pode não corresponder aos desejos e preferências alimentares dos escolares, assim como a prevalência de estudantes que consomem outros alimentos (alimentos competidores) durante o período que permanecem na escola (Paiva, Freitas \& Santos, 2016).

Apesar de o consumo alimentar dos adolescentes mostrar-se caloricamente inadequado, observou-se que o RA está dentro da faixa, mas tendendo ao limite inferior, enquanto no QFA verificou-se consumo acima do limite superior ao recomendado pela IOM (2002/2005), ou seja, a maioria dos adolescentes apresentaram valores de consumo energético nos limites inferiores ou superiores ao proposto pela EER. Minuzzi, Lara \& Coutinho, (2020) em seu estudo constataram dados que se aproximam dos aqui apresentados, com consumo médio de 2.903,74 (IC95\%: 2024,04 - 3783,44) kcal diárias, com os meninos apresentando maior ingestão calórica total (3549,82 kcal, IC95\%: 2026,09 - 5073,54).

Além das limitações que os próprios instrumentos de avaliação de consumo apresentam como o QFA e o próprio instrumento de referência utilizado - RA, outras podem ser apontadas: com a prática clínica é possível observar um hábito comum aos entrevistados por métodos de estimação do consumo alimentar, a exemplo dos que foram utilizados no estudo, quando as pessoas são observadas ou questionadas a respeito do que comem, elas tendem a modificar o seu padrão alimentar, bem como, hábitos e atitudes, em relação aos alimentos, isso pode contribuir para que ocorram vieses de informação levando a superestimação ou subestimação do real consumo alimentar. Para minimizar esses vieses, o QFA foi aplicado antes do instrumento de referência (Cade et al., 2004), e um estudo piloto em Salvador/Bahia testou o instrumento com adolescentes escolares de uma escola pública não incluída na amostra, possibilitando a revisão do quantitativo de itens e a abordagem com os escolares. Quanto ao RA, às orientações para o preenchimento foram acompanhadas por álbum com porções de alimentos e de utensílios, e a equipe de campo estava disponível para retirada de dúvidas durante a entrega dos registros. Outra forma para minimizar os erros advindos dos RA não foi utilizada devido a dificuldades materiais e de logística, a exemplo do uso de balanças para avaliar o tamanho das porções, eliminando a dependência da percepção das quantidades ingeridas dos alimentos (Willett, 2013), o que pode caracterizar uma limitação do estudo. Entretanto, o registro dietético de três dias, sendo dois dias da semana e um dia do final de semana, permite aferir a variabilidade do consumo alimentar e minimizar possíveis vieses. 
Outras implicações recaem sobre as equações preditivas, que são métodos bastante utilizados para a estimativa de energia, porém, requerem a estimativa do gasto energético das atividades físicas realizadas durante o dia, o que implica a necessidade de autorrelato sobre a atividade física diária, fato que pode contribuir para que ocorram erros nas interpretações de dados. É importante destacar que as DRIs foram estabelecidas para a população norte-americana, e para sua utilização na população brasileira devem se considerar prováveis diferenças e, consequentemente, alguns erros associados (Melo, Tirapegui \& Ribeiro, 2008). Porém, o IPAQ, que fornece informações sobre o dispêndio energético e utilizado neste estudo, foi validado para o Brasil (Matsudo et al., 2001) e tem sido amplamente utilizado em estudos populacionais.

E por fim, o número amostral ficou limitado em função do quantitativo de respondentes aos registros, porém, foram envolvidos adolescentes escolares de dez escolas públicas em dez municípios baianos. Acredita-se que o estudo tem potencial para inspirar novas pesquisas com esse grupo populacional, ao evidenciar diferenças entre as necessidades energéticas calculadas por modelos matemáticos e os dados obtidos por meio de dois instrumentos de avaliação de consumo alimentar, para a população do estudo, que pode se estender aos demais participantes do PROSE.

Este estudo pode contribuir com questões importantes sobre a saúde e estilo de vida de adolescentes escolares, primeiramente ao identificar comportamentos que são reconhecidamente protetores à saúde, relacionados a hábitos alimentares e de atividade física, diferindo do esperado para esse grupo etário que comumente apresenta comportamentos de risco como pular refeições, sedentarismo e alimentar-se fora de casa. Outra contribuição é metodológica e vem do uso de dois instrumentos amplamente utilizados para avaliação do consumo, sendo um deles já validado para a população do estudo. A população deste estudo é oriunda de municípios de pequeno porte do interior baiano e a maioria dos estudos é realizada na capital ou grandes cidades (Neves, Gonzaga, Martens, \& Moura., 2010; Araki et al., 2011; Rodrigues et al., 2012; Marchioni, et al., 2015). Estudar o consumo alimentar e as necessidades energéticas de adolescentes se mostrou um desafio considerando tanto as características próprias dessa população quanto as dificuldades em se mensurar construtos complexos.

\section{Considerações Finais}

Ao comparar as recomendações internacionais com o consumo de adolescentes escolares do interior baiano, verificouse discrepâncias entre o recomendado e o consumido, em relação ao consumo energético. Entretanto, apesar dessa inadequação percebeu-se que a população do estudo tem baixa frequência dos extremos do estado nutricional (magreza ou sobrepeso e obesidade). Entende-se que o nível de atividade física encontrado nessa população pode estar intervindo nessa relação. Assim, sugere-se que os futuros estudos foquem em análises que avaliem o papel da atividade física na mediação da relação do consumo alimentar e estado nutricional.

A avaliação das necessidades nutricionais estimadas para grupos populacionais é de importância no âmbito da Saúde Pública, bem como, conhecer a sua situação alimentar e nutricional atual. Esse conjunto de análises permite entender os desvios existentes e a proposição de ações que busquem promover a saúde dos indivíduos, em especial, os indivíduos em estágios de vida críticos como os adolescentes.

\section{Agradecimentos}

Aos componentes do Programa Integrado de Pesquisa e Cooperação Técnica em Formação e Avaliação da Atenção Básica (GRAB), aos dirigentes escolares que apoiaram o Prose, aos adolescentes escolares que participaram da pesquisa, ao Programa Permanecer da Pró-Reitoria de Assistência Estudantil (PROAE) da UFBA pela concessão de bolsa auxílio aos graduandos da pesquisa e a prof ${ }^{a}$, dra. Maria da Purificação Nazaré Araújo pela prestatividade na execução do artigo. O presente estudo é fruto do trabalho de conclusão de curso (TCC) apresentado a Escola de Nutrição da UFBA. 


\section{Referências}

Ambrosini, G. L., Oddy, W. H., Robinson, M., O’Sullivan, T. A., Hands, B. P., De Klerk, N. H., \& Beilin, L. J. (2009). Adolescent dietary patterns are associated with lifestyle and family psycho-social factors. Public health nutrition, 12(10), 1807-1815.

Araki, E. L., Philippi, S. T., Martinez, M. F., Estima, C. D. C. P., Leal, G. V. S., \& Alvarenga, M. D. S. (2011). Padrão de refeições realizadas por adolescentes que frequentam escolas técnicas de São Paulo. Revista Paulista de Pediatria, 29, 164-170.

Aranceta, J., Perez-Rodrigo, C., Ribas, L., \& Serra-Majem, L. L. (2003). Sociodemographic and lifestyle determinants of food patterns in Spanish children and adolescents: the enKid study. European Journal of Clinical Nutrition, 57(1), S40-S44.

Araújo, C., Toral, N., Silva, A. C. F. D., Velásquez-Melendez, G., \& Dias, A. J. R. (2010). Estado nutricional dos adolescentes e sua relação com variáveis sociodemográficas: Pesquisa Nacional de Saúde do Escolar (PeNSE), 2009. Ciência \& Saúde Coletiva, 15, $3077-3084$.

Ashwell, M., \& Hsieh, S. D. (2005). Six reasons why the waist-to-height ratio is a rapid and effective global indicator for health risks of obesity and how its use could simplify the international public health message on obesity. Int J Food Sci Nutr. 56, 303-307.

Barquera, S., Hernandez-Barrera, L., Tolentino, M. L., Espinosa, J., Ng, S. W., Rivera, J. A., \& Popkin, B. M. (2008). Energy intake from beverages is increasing among Mexican adolescents and adults. The Journal of nutrition, 138(12), 2454-2461.

Benedetti, T. R. B., Antunes, P. D. C., Rodriguez-Añez, C. R., Mazo, G. Z., \& Petroski, É. L. (2007). Reprodutibilidade e validade do Questionário Internacional de Atividade Física (IPAQ) em homens idosos. Revista Brasileira de Medicina do Esporte, 13, 11-16.

Berz, J. P., Singer, M. R., Guo, X., Daniels, S. R., \& Moore, L. L. (2011). Use of a DASH food group score to predict excess weight gain in adolescent girls in the National Growth and Health Study. Archives of pediatrics \& adolescent medicine, 165(6), 540-546.

Bezerra, I. N., \& Sichieri, R. (2010). Características y gastos con alimentación fuera del domicilio en Brasil. Revista de Saúde pública, 44(2), 221-229.

Bland, J. M. (2004). Cluster randomised trials in the medical literature: two bibliometric surveys. BMC medical research methodology, 4(1), 1-6.

Brito, A. P., Araujo, M. C., Guimarães, C. P., \& Pereira, R. A. (2017). Validade relativa de questionário de frequência alimentar com suporte de imagens. Ciência \& Saúde Coletiva, 22, 457-468.

Cade, J., Thompson, R., Burley, V., \& Warm, D. (2002). Development, validation and utilisation of food-frequency questionnaires-a review. Public health nutrition, 5(4), 567-587.

Costa, C. D. S., Flores, T. R., Wendt, A., Neves, R. G., Assunção, M. C. F., \& Santos, I. S. (2018). Comportamento sedentário e consumo de alimentos ultraprocessados entre adolescentes brasileiros: Pesquisa Nacional de Saúde do Escolar (PeNSE), 2015. Cadernos de Saúde Pública, 34.

Cruciani, F., Araújo, T., Matsudo, S., \& Matsudo, V. (2009). Nível de atividade física de mulheres maiores de 50 anos de idade participantes de um programa de atividade física estruturada. Rev. bras. ciênc. mov, 1-16.

De Souza Lima, R., Neto, J. A. F., \& Farias, R. D. C. P. (2015). Alimentação, comida e cultura: o exercício da comensalidade. DEMETRA: Alimentação, Nutrição \& Saúde, 10(3), 507-522.

De Souza, J. B., \& ENES, C. C. (2013). Influência do consumo alimentar sobre o estado nutricional de adolescentes de Sorocaba-SP.

De Souza, S., Marques, K. C., \& Reuter, C. P. (2020). Tempo de tela acima das recomendações em crianças e adolescentes: análise dos fatores nutricionais, comportamentais e parentais associados. Journal of Human Growth and Development, 30(3), 363.

del Mar Bibiloni, M., Pich, J., Córdova, A., Pons, A., \& Tur, J. A. (2012). Association between sedentary behaviour and socioeconomic factors, diet and lifestyle among the Balearic Islands adolescents. BMC Public Health, 12(1), 1-11.

Farias Júnior, J. C. D., Nahas, M. V., Barros, M. V. G. D., Loch, M. R., Oliveira, E. S. A. D., De Bem, M. F. L., \& Lopes, A. D. S. (2009). Comportamentos de risco à saúde em adolescentes no Sul do Brasil: prevalência e fatores associados. Revista Panamericana de Salud Pública, 25, 344-352.

Fisberg, R. M., Slater, B., Marchioni, D. M. L., \& Martini, L. A. (2005). Inquéritos alimentares: métodos e bases científicos. In Inquéritos alimentares: métodos e bases científicos (pp. 334-334).

Fonseca, R. D. R. Estudos sobre padrões alimentares na adolescência: contribuições teórico-metodológicas, 2017. Tese (doutorado) - Programa de PósGraduação em Saúde Coletiva, Instituto de Saúde Coletiva. Universidade Federal da Bahia.

Fonseca-Barroso, R. D. R. F., Aquino, R., \& Amorim, L. D. A. F. (2021). Consumo alimentar de adolescentes: Validação e calibração de um questionário de frequência alimentar em estudo com amostragem complexa. Research, Society and Development, 10(6), e5391016075-e5391016075.

Freedman, D. S., Serdula, M. K., Srinivasan, S. R., \& Berenson, G. S. (1999). Relation of circumferences and skinfold thicknesses to lipid and insulin concentrations in children and adolescents: the Bogalusa Heart Study. The American journal of clinical nutrition, 69(2), 308-317.

Henry, C. J. K. (2005). Basal metabolic rate studies in humans: measurement and development of new equations. Public health nutrition, 8(7a), 1133-1152

Instituo Brasileiro de Geografia e Estatística, BRASIL. (2011). Pesquisa de orçamentos familiares 2008-2009: Análise do consumo alimentar pessoal no Brasil. IBGE. www.ibge.gov.br.

Instituo Brasileiro de Geografia e Estatística, BRASIL. (2020). Pesquisa de Orçamentos Familiares 2017-2018. Avaliação Nutricional da Disponibilidade Domiciliar de Alimentos no Brasil. IBGE. www.ibge.gov.br. 
International Diabetes Federation - IDF. (2007). The IDF consensus definition of the Metabolic Syndrome in children and adolescents 2007. http://www.idf.org/home / http://www.idf.org/metabolic-syndrome/children/criteria Acesso em 24102021.

Institute of Medicine - IOM. (2002/2005). DRIs - Dietary Reference Intakes for energy, carbohydrate, fiber, fat, fatty acids, cholesterol, protein, and amino acids. Washington, D.C.: National Academy Pres. https://www.ncbi.nlm.nih.gov/pubmed/12449285.

IPAQ Research Committee. (2005). Guidelines for data processing and analysis of the International Physical Activity Questionnaire (IPAQ)-short and long forms. http://www. ipaq. ki. se/scoring. pdf.

Köche, J. C. (2011). Fundamentos de metodologia científica: teoria da ciência e iniciação à pesquisa. Vozes. http://www.adm.ufrpe.br/sites/ww4.deinfo.ufrpe.br/files/Fundamentos_de_Metodologia_Cienti\%CC\%81fica.pdf.

Lake, A. A., Mathers, J. C., Rugg-Gunn, A. J., \& Adamson, A. J. (2006). Longitudinal change in food habits between adolescence (11-12 years) and adulthood (32-33 years): the ASH30 Study. Journal of public health, 28(1), 10-16.

Leal, G. V. D. S., Philippi, S. T., Matsudo, S. M. M., \& Toassa, E. C. (2010). Consumo alimentar e padrão de refeições de adolescentes, São Paulo, Brasil. Revista Brasileira de Epidemiologia, 13, 457-467.

Levy, R. B., Castro, I. R. R. D., Cardoso, L. D. O., Tavares, L. F., Sardinha, L. M. V., Gomes, F. D. S., \& Costa, A. W. N. D. (2010). Consumo e comportamento alimentar entre adolescentes brasileiros: Pesquisa Nacional de Saúde do Escolar (PeNSE), 2009. Ciência \& Saúde Coletiva, 15, $3085-3097$.

Lima, N. M. D. S., Leal, V. S., Oliveira, J. S., Andrade, M. I. S. D., Tavares, F. C. D. L. P., Menezes, R. C. E. D., \& Lira, P. I. C. D. (2017). Excesso de peso em adolescentes e estado nutricional dos pais: uma revisão sistemática. Ciência \& Saúde Coletiva, 22, 627-636.

Lohman, T. G., Roche, A. F., \& Martorell, R. (1988). Anthropometric standardization reference manual. ed. Champaign IL: Human Kinetics Books.

Marchioni, D. M. L., Gorgulho, B. M., Teixeira, J. A., Verly Junior, E., \& Fisberg, R. M. (2015). Prevalência de omissão do café da manhã e seus fatores associados em adolescentes de São Paulo: estudo ISA-Capital. Nutrire Rev. Soc. Bras. Aliment. Nutr, 10-20.

Mascarenhas, J. M. O., Silva, R. D. C. R., Assis, A. M. O. D., Santana, M. L. P. D., Moraes, L. T. L. P. D., \& Barreto, M. L. (2014). Identification of food intake patterns and associated factors in teenagers1. Revista de Nutrição, 27, 45-54.

Matsudo, S., Araújo, T., Matsudo, V., Andrade, D., Andrade, E., \& Oliveira, L. C. (2001). Questionário Internacional de Atividade Fisica (IPAQ): Estudo de validade e reprodutibilidade no Brasil. Atividade Física e Saúde 6 (2): 5-18.

Melo, C. M. D., Tirapegui, J., \& Ribeiro, S. M. L. (2008). Gasto energético corporal: conceitos, formas de avaliação e sua relação com a obesidade. Arquivos Brasileiros de Endocrinologia \& Metabologia, 52, 452-464.

Mendonça, S. K., de Carvalho, A. P., de Oliveira Assis, D. F., \& Moreira, D. C. (2019). Prevalência de excesso de peso em adolescentes de escolas quilombolas e os fatores associados. RBONE-Revista Brasileira de Obesidade, Nutrição e Emagrecimento, 13(81), 750-758.

Minuzzi, M. D., Lara, S., \& Coutinho, R. X. (2020). Hábitos alimentares de adolescentes estudantes de um instituto federal do Estado do Rio Grande do Sul, Brasil. Research, Society and Development, 9(9), e45997054-e45997054.

Monge-Rojas, R. (2001). Dietary intake as a cardiovascular risk factor in Costa Rican adolescents. Journal of adolescent health, $28(4), 328-337$.

Monteiro, L. S., Hassan, B. K., Estima, C. C. P., Souza, A. D. M., Verly, E., Sichieri, R., \& Pereira, R. A. (2017). Consumo alimentar segundo os dias da semana-Inquérito Nacional de Alimentação, 2008-2009. Revista de Saúde Pública, 51.

Moreno, L. A., Gottrand, F., Huybrechts, I., Ruiz, J. R., González-Gross, M., DeHenauw, S., \& HELENA Study Group. (2014). Nutrition and lifestyle in european adolescents: the HELENA (Healthy Lifestyle in Europe by Nutrition in Adolescence) study. Advances in Nutrition, 5(5), 615S-623S.

Neves, A. C. M. D., Gonzaga, L. A. A., Martens, I. B. G., \& Moura, E. C. (2010). Validação de indicadores do consumo de alimentos e bebidas obtidos por inquérito telefônico em Belém, Pará, Brasil. Cadernos de Saúde Pública, 26, 2379-2388.

Oehlschlaeger, M. H. K., Pinheiro, R. T., Horta, B., Gelatti, C., \& San'Tana, P. (2004). Prevalência e fatores associados ao sedentarismo em adolescentes de área urbana. Revista de Saúde Pública, 38, 157-163.

Paiva, J. B. D., Freitas, M. D. C. S. D., \& Santos, L. A. D. S. (2016). Significados da alimentação escolar segundo alunos atendidos pelo Programa Nacional de Alimentação Escolar. Ciência \& Saúde Coletiva, 21, 2507-2516.

Pereira, P. F., Serrano, H. M. S., Carvalho, G. Q., Lamounier, J. A., Peluzio, M. D. C. G., Franceschini, S. D. C. C., \& Priore, S. E. (2011). Circunferência da cintura e relação cintura/estatura: úteis para identificar risco metabólico em adolescentes do sexo feminino. Revista Paulista de Pediatria, 29(3), 372-7. <http://www.scielo.br/scielo.php?script=sci_arttext\&pid=S0103-05822011000300011\&lng=en\&nrm=iso>.

Política Nacional de Alimentação e Nutrição. (2013). Ministério da Saúde, Secretaria de Atenção à Saúde. Departamento de Atenção Básica.Básica. Ministério da Saúde.

Richmond, T. K., \& Subramanian, S. V. (2008). School level contextual factors are associated with the weight status of adolescent males and females. Obesity, 16(6), 1324-1330.

Rodrigues, P. R. M., Pereira, R. A., Cunha, D. B., Sichieri, R., Ferreira, M. G., Vilela, A. A. F., \& Gonçalves-Silva, R. M. V. (2012). Fatores associados a padrões alimentares em adolescentes: um estudo de base escolar em Cuiabá, Mato Grosso. Revista Brasileira de Epidemiologia, 15, 662-674.

Santos, N. H. A. D., Fiaccone, R. L., Barreto, M. L., Silva, L. A. D., \& Silva, R. D. C. R. (2014). Association between eating patterns and body mass index in a sample of children and adolescents in Northeastern Brazil. Cadernos de saude publica, 30, 2235-2245. 
Research, Society and Development, v. 10, n. 14, e373101422275, 2021

(CC BY 4.0) | ISSN 2525-3409 | DOI: http://dx.doi.org/10.33448/rsd-v10i14.22275

Schneider, B. C., Motta, J. V. D. S., Muniz, L. C., Bielemann, R. M., Madruga, S. W., Orlandi, S. P., \& Assunção, M. C. F. (2016). Desenho de um questionário de frequência alimentar digital autoaplicado para avaliar o consumo alimentar de adolescentes e adultos jovens: coortes de nascimentos de Pelotas, Rio Grande do Sul. Revista Brasileira de Epidemiologia, 19, 419-432.

Schofield, W. N. (1985). Predicting basal metabolic rate, new standards and review of previous work. Human nutrition. Clinical nutrition, 39, 5-41.

Silva, E. O., Amparo-Santos, L., \& Soares, M. D. (2018). Alimentação escolar e constituição de identidades dos escolares: da merenda para pobres ao direito à alimentação. Cadernos de Saúde Pública, 34.

Simões, A. M., Machado, C. O., \& Höfelmann, D. A. (2021). Associação do consumo regular de café da manhã e comportamentos relacionados à saúde em adolescentes. Ciência \& Saúde Coletiva, 26, 2243-2251.

Trancoso, S. C., Cavalli, S. B., \& Proença, R. P. D. C. (2010). Café da manhã: caracterização, consumo e importância para a saúde. Revista de Nutrição, 23, 859-869.

Valentim, E. D. A., Almeida, C. C. B. D., Taconeli, C. A., Osório, M. M., \& Schmidt, S. T. (2017). Fatores associados à adesão à alimentação escolar por adolescentes de escolas públicas estaduais de Colombo, Paraná, Brasil. Cadernos de Saúde Pública, 33, e00061016.

Vasconcelos, M. J. O. B., Barbosa, J. M., Pinto, I. D. S., Lima, T. D., \& Araújo, A. D. (2011). Nutrição clínica: obstetrícia e pediatria. Medbook.

Voci, S. M., Enes, C. C., \& Slater, B. (2008). Validação do Questionário de Freqüência Alimentar para Adolescentes (QFAA) por grupos de alimentos em uma população de escolares. Revista Brasileira de Epidemiologia, 11, 561-572.

Willett, W. C. (2013). Nutritional epidemiology. Oxford University Press, (3a ed.), vol. 40.

World Health Organization. (2007a) Growth reference 5-19 years. Geneva, 2007. 〈http://www.who.int/growthref/who2007_bmi_for_age/en/>

World Health Organization. (2007b) Growth reference 5-19 years. Geneva, 2007. <http://www.who.int/growthref/who2007_bmi_for_age/en/>

World Health Organization. (2013). Global action plan for the prevention and control of noncommunicable diseases 2013-2020. < https://www.who.int/publications-detail-redirect/9789241506236>

World Health Organization. WHO Maternal, Newborn, Child and Adolescent Health and Ageing Data Portal. Adolescent - Risk factors. https://www.who.int/data/maternal-newborn-child-adolescent-ageing/adolescent-data/adolescent---risk-factors

World Health Organization. (2005). Nutrition in adolescence: issues and challenges for the health sector: issues in adolescence health and development. Genova: WHO. <http://apps.who.int/iris/bitstream/10665/43342/1/9241593660_eng.pdf?ua=1> 